\title{
Use of Telemedicine in Postgraduate Pathology Education*
}

\author{
Asaranti Kar\#, Tushar Kar, Priyadarshini Biswal, Kaumudee Pattanaik, Pallavi Bhuyan, \\ Rajashree Mallick, B. N. Mohanty
}

Department of Pathology, Shriramachandra Bhanj Medical College, Cuttack, Odisha, Pin-753007, India.

Email: \#asarantikar@yahoo.co.in

Received March 28 ${ }^{\text {th }}, 2013$; revised May $18^{\text {th }}, 2013$; accepted May $26^{\text {th }}, 2013$

Copyright (C) 2013 Asaranti Kar et al. This is an open access article distributed under the Creative Commons Attribution License, which permits unrestricted use, distribution, and reproduction in any medium, provided the original work is properly cited.

\begin{abstract}
Objective: Telemedicine is the use of telecommunication for medical diagnosis, treatment, health education and research. Telepathology is diagnostic pathology at a distance. It functions with images viewed on a video monitor rather than directly through a microscope. Many years have passed since the evolution of telepathology. But no study has yet been done to evaluate the benefits postgraduates got through this. Therefore this study is undertaken to analyse the different impacts of telepathology on the postgraduate learning. Material and Methods: The study was done in Telemedicine Center of S. C. B. Medical College, Cuttack over a period of 9 years. The telepathology service was carried out by store and forward method using a CCD, a large computer server and a huge bandwidth network service (VSAT). The sessions with SGPGI, Lucknow were analysed and the benefits of postgraduates were studied. Results: The number of sessions held was 95. Out of which 92 sessions were with SGPGI. It included 202 cases transmitted from Lucknow and 186 cases from S. C. B. Medical College, Cuttack. The postgraduates had a chance to exposure to rare cases in renal pathology, dermatopathology and hepatopathology. The overall concordance rate with glass slide diagnosis was $87 \%$. Conclusion: The diagnostic expertise through light microscopy and tele-images are not the same. But the telepathology system increased the ability of image analysis, histopathologic diagnosis of our postgraduates and also exposed them to many rare and interesting cases.
\end{abstract}

Keywords: Histopathologic Diagnosis; Postgraduates; Telepathology

\section{Introduction}

Telemedicine primarily refers to use of telecommunication for diagnosis and treatment of diseases. Telepathology is one of the applications of telemedicine. It uses technology to facilitate the transfer of image-rich pathology data between distant locations for the purposes of diagnosis, education, and research. [1,2] Telepathology, literally meaning practice of pathology at a distance, was first coined by the academic pathologist Ronald S. Winstein, MD in 1986 in a medical journal editorial. [3] He is known by many as the "Father of Telepathology". Since its inception, it has successfully been implemented in many countries of Europe, America and Asia. In India, it has been in use in telemedicine centres like PGI, Chandigarh, SGPGI, Lucknow, AIIMS, New Delhi, TMH, Mumbai and many centers including S. C. B. Medical College, Cuttack. Since 2003 till today, there have been

\footnotetext{
*There is no commercial association that might create conflict of interest in connection with the submitted manuscript.

\#Corresponding author.
}

many sessions with other centres in India from S. C. B. Medical College imbibing knowledge from experts from outside the state and disseminating medical information to different telemedicine centers throughout the state.

But since 1986 till 2012, no study has been undertaken to analyze the usefulness of telemedicine in postgraduate education. The exposure of postgraduates to difficult and the rare yet interesting cases are observed and also acquaintance with diagnosis which is not made often in our institution was seen. Therefore this study is performed to see the role of telepathology in enabling the postgraduates to correct histopathological diagnosis of difficult and controversial cases.

\section{Material and Methods}

The study was conducted in Telemedicine Center, Department of Pathology, S. C. B. Medical College, Cuttack, Odisha. The basic principle is the acquisition of histological and/or macroscopic images for transmission along telecommunication pathways for diagnosis, con- 
sultation and continuing medical education. A telepathology system comprises of a conventional microscope, a method of image capture, commonly a camera mounted on a light microscope, telecommunication link between sending and receiving sites, and a workstation at the receiving site with a high quality monitor to view the images. The telepathology service functions here with a charge couple device scanner (slide scanner-CCD or digital snap shot camera) to receive and convert the images into electronic form, a large computer server and huge bandwidth network links (VSAT). This service was made available in this medical college since 2003 and is successfully conducted every month. Mostly the telepathology service is carried out by static imaging method (store and forward approach) or rarely with live video images. The case history, investigation findings, gross picture and 3 to 8 photomicrographic images at different magnifications are transferred before time. Following the transmission of images the interpretation of cases and discussion about final diagnosis are carried out by videoconferencing at both ends. The impression from pathologists of both sides is analyzed and after the ancillary studies, the final diagnosis is reached at. Most of the times the glass slides are sent to another pathology center and the final diagnosis is received. The histopathologic diagnoses from both sides are correlated.

\section{Results}

The study was conducted over a period of 9 years from January, 2003 till December, 2012. The different telemedicine programs are carried out in the Telemedicine seminar hall of S. C. B. Medical College according to the monthly schedule (Table 1). Total 95 sessions of telepathology were carried out. Out of them, 3 sessions were with All India Institute of Medical Sciences (AIIMS) New Delhi, 92 were with Sanjay Gandhi Post Graduate Institute, Lucknow (SGPGI). This includes 37 sessions transmitted from SGPGI and 45 were from S. C. B. Medical College, Cuttack. The sessions from SGPGI were educative where they have already reached at the diagnosis and comprise of 202 interesting cases. In total, 740 images were transmitted for this. The majority of these belong to renal pathology (148 cases or $73.3 \%$ ) followed by dermatopathology and hepatology (Table 2). Renal cases were mostly non-neoplastic $(116 \%$ or $78.4 \%$ cases) including majority of the posttransplant complications (80\%/54.1\%) (Table 3). The cases from dermatopathology were mostly vesiculo-bullous lesions followed by mycotic lesions and the neoplastic cases were mostly benign and malignant appendageal tumours. The sessions from SCB Medical College were consultative type where the final diagnosis was not known because of lack of facility for ancillary studies. This comprise of 186 cases with transmission of 692 images. Most of the cases were
Table 1. Distribution of telemedicine sessions in a month in S. C. B. Medical College, Cuttack.

\begin{tabular}{ccc}
\hline $\begin{array}{c}\text { Branch of } \\
\text { telemedicine }\end{array}$ & $\begin{array}{c}\text { No. of sessions } \\
\text { per month }\end{array}$ & $\begin{array}{c}\text { \% of sessions } \\
\text { per month }\end{array}$ \\
\hline Medicine & 01 & 05.3 \\
Pulmonary medicine & 02 & 10.5 \\
Hepatology & 02 & 10.5 \\
Surgery & 02 & 10.5 \\
GU surgery & 01 & 05.3 \\
Neurosurgery & 01 & 05.3 \\
Endocrine surgery & 03 & 15.8 \\
Obstetrics \& gynecology & 03 & 15.8 \\
Pathology & 03 & 15.8 \\
Microbiology & 01 & 05.3 \\
\hline
\end{tabular}

Table 2. Distribution of cases from SGPGI, Lucknow ( $n=$ 202).

\begin{tabular}{cccc}
\hline System & Total no. of cases & Neoplastic & Non-neoplastic \\
\hline Renalpathology & $148(73.3 \%)$ & $32(21.6 \%)$ & $116(78.4 \%)$ \\
Dermatopathology & $34(16.8 \%)$ & $16(47.0 \%)$ & $18(53.0 \%)$ \\
Hepatopathology & $20(09.9 \%)$ & $08(40 \%)$ & $12(60 \%)$ \\
\hline
\end{tabular}

Table 3. Distribution of renal cases $(n=148)$.

\begin{tabular}{|c|c|c|}
\hline $\begin{array}{l}\text { Post-transplant } \\
\text { complications }\end{array}$ & Glomerulonephritis & Neoplasms \\
\hline Infectious Non-infectious & & Benign Malignant \\
\hline $58(39.2 \%) \quad 22(14.9 \%)$ & $36(24.3 \%)$ & 09 (6.0\%) 23 (15.6\%) \\
\hline
\end{tabular}

from central nervous system (62 cases or 33.3\%) (Table 4). Out of the consultative cases the diagnostic correlation was $87 \%$ (162/186 cases) between glass slides and telepathology impression from SGPGI, Lucknow.

\section{Discussion}

Telepathology is the latest development in medical science and a very important asset in health care system. It differs from other branches of telemedicine in which telepathology deals with images instead of live patients. The original specimen here is a slide. And it also needs to convert the images into digital form. This leads to loss of quality in the images. Digitalization of all the images in different magnifications can lead to a large amount of data which can be difficult to transmit and also to view. Moreover, there are no international standards and technical specifications to follow as gold standard in telepathology. Undoubtedly, it is a boon to the pathologists in smaller laboratories or hospitals in India who come across unconventional or controversial cases during their routine practice. For the budding pathologists like the postgraduate students it can act as an excellent tool for learning and gaining exposure to variety of cases. 
Table 4. Distribution of cases from S. C. B. Medical College, Cuttack $(n=186)$.

\begin{tabular}{ccc}
\hline System & No. of cases & \% of cases \\
\hline CNS & 62 & 33.3 \\
GIT & 22 & 11.8 \\
Endocrine & 17 & 09.2 \\
Female genital tract & 09 & 04.8 \\
Soft tissue & 23 & 12.4 \\
Lymphoreticular & 05 & 02.7 \\
Breast & 03 & 01.6 \\
Bone & 10 & 05.4 \\
Skin & 11 & 05.9 \\
Testis & 06 & 03.2 \\
Mediastinum & 07 & 03.8 \\
Salivary gland & 10 & 05.4 \\
Eye lid & 01 & 00.5 \\
\hline
\end{tabular}

There are three telepathology systems: static imagebased systems, real time systems and virtual slide systems. Static image system is the most reasonably priced and usable system. But it can only capture selected microscopic fields for off-site evaluation. With real time systems, the consultant pathologist operates a robotically controlled motorized microscope located at a distant site changing focus and field of view at will. Virtual slide system creates a digital image file of an entire glass slide (whole slide image) utilizing digital slide scanners. This file is stored on a computer server and can be navigated at a distance, over the internet, using a browser. [4] Depending on the management and interactivity of images, telepathology is again divided into: 1) Static telepathology (Teleconsultation): done with Static Images sent for consultation in various ways (ftp, www, static images under videotelephony, modem, etc.); 2) Kinetic telepathology: that includes the capability to monitor the microscope at distance in order to do the sampling. Images are sent in full resolution either as static images, as live highly compressed images (i.e. through videoconferencing standards) or both at the same times (i.e. for Intraoperative specimens); 3) Dynamic telepathology: that includes with the 2) option, and the capability of having full colour non-compressed images in real time (Live).

In our telemedicine centre, we use mostly the static image based system (store and forward method) for transmission of study material. While real-time and virtual slide systems offer higher diagnostic accuracy when compared with static-image telepathology, there are drawbacks to each. Real-time systems perform best on local area networks (LANs), but performance may suffer if it is employed during periods of high network traffic or using the Internet proper as a backbone. Expense is an issue with real-time systems and virtual slide systems as they can be costly.

Since 2003, when the telemedicine network was established in S. C. B. Medical College, there had been one international conference, one national conference, 12 CMEs and many monthly seminars. The monthly telemedicine activities are conducted according to the monthly schedule which is circulated in all the departments before hand. It includes three sessions, each of telepathology, obstetrics and gynecology, and endocrine surgery (Table 1).

Telepathology is already integrated into histopathology services in many countries. It mostly deals with histopathology along with cytology and hematology. But histopathology is a discipline where the importance of consultation with colleagues, expert second opinions, and collective postgraduate education is highly felt. Images of tissue viewed through microscope form the primary diagnostic medium for diagnosis. At present, the most common method of sharing images is by sending the glass slides themselves. [5] But the electronic capture and distribution of images is an alternative, easy, fast and more efficient system. The operational efficacy of telepathology, videoconferencing and digital imaging is under evaluation in several different clinical contexts at present. [6,7] In the present study, the cases transmitted from SGPGI included mostly the systems to which the postgraduates were underexposed like renal pathology and others (Table 2). Especially the post-transplant complication cases were very informative which exposed our students to infectious pathology like fungal infections, cytomegalovirus inclusions and microfilarial infestation. The different varieties of glomerulonephritis cases were associated with the findings of special stains like Periodic Acid Schiff (PAS), Silver methylamine and also immunofluoresence pictures. There were all the variants of focal segmental glomerulonephritis (FSGS) and the accompanying discussion which were highly educative for the students. Renal pathology slides shown included majority of post-transplant complications (54.1\%) followed by glomerulonephritis.

But the cases of our institution comprised mostly of central nervous system (33.3\%) followed by soft tissue tumours (23 cases/12.4\%). The percentage of correlation was maximum in central nervous system (Table 4). The cases from central nervous system were mostly neoplastic and associated with squash cytology findings and the discussion through videoconferencing was encouraging for the postgraduate students. The percentage of correlation was highest (92\%) with lesions of central nervous system. The correlation was lowest (80\%) with soft tissue lesions because of lack of exposure to more of such cases and also due to absence of ancillary studies.

It is difficult to assess the accuracy of telepathology. 
Here after the diagnosis was obtained from the telepathology center of SGPGI, Lucknow the slides were sent to a referral center or to a referral pathologist or the ancillary methods of diagnosis like immunohistochemistry were adopted. In all the situations a final diagnosis was obtained in each case. The diagnostic accuracy between glass slides and SGPGI was 87\% (in 162 cases out of 186 cases). This finding correlates with that of Shimosato et $a l$. who had reported a concordance of $88.1 \%$ based upon findings of one pathologist. [8] Winberg et al. found overall diagnostic accuracy of $85.5 \%$ when used four pathologists at Brigham and Women's Hospital in Boston. They were shown 50 static images from selected cases and were given the conventional glass slides at a later date. Both the opinions were compared. [9] It is difficult to ascertain whether it depends on the technique or the skill of the histopathologist. However the concordance was $100 \%$ when discriminating benign from malignant lesions while minor discripancies relating to precise characterization persisted. Work published from Arizona-International Telemedicine Network divided the reasons for discordance into three categories: field selection, diagnostic interpretation and video image quality. [10] Diagnostic interpretation skill was sited with the most important reason followed by field selection.

In our study, the correlation was more where the gross specimen was smaller not having a variegated appearance where the operator has to look into the gross himself, images were of good quality and some ancillary studies like special stains, immunohistochemistry done and the investigations were complete. The causes of non-correlation are large specimens with variegated appearance, thick section and poor images out of focus, lack of ancillary studies, borderline malignant cases without adequate number of representative images. Therefore the uniform standards for capturing, archiving and transferring images in telepathology protocol need to be developed for effective telepathology practice.

Benefits for postgraduates: telepathology is currently being used for a wide spectrum of clinical applications including diagnosis of frozen section specimens, [11] primary histopathology diagnoses [12], second opinion diagnoses, [13] subspecialty pathology expert diagnoses, [14] education, [15] compentency assessment, [16] and research. Benefits of telepathology include providing immediate access to off-site pathologists for rapid frozen section diagnoses. Another benefit can be gained direct access to subspecialty pathologists such as a renal pathologist, a neuropathologist, or a dermatopathologist, for immediate consultations. In the present study the postgraduates could acquire knowledge in renal pathology, and frozen section specimens which were recently introduced in our college.

Most histopathologists do not routinely use or have access to a telepathology workstation and hence they can not use it in daily diagnosis. But the postgraduate students of our institute have learnt it and can use it when needed. It was observed that the young postgraduates became more familiar with the digital images and had increased diagnostic accuracy compared to the older students.

The students could access the special diagnostic services like transplant related pathology, ophthalmic pathology, dermatopathology, neuropathology etc. Instant access to international expertise could be possible with the limited human resources.

Demerits: however, the ultimate role of telepathology is uncertain. More evaluation and education are essential in this field. To nearly all the pathologists working without a microscope are unconceivable. The expertise for diagnosis through light microscope and tele-images are not the same. If the originating pathologist (sender) misses an area on the glass slide of potential diagnostic importance, so will the receiving or consulting pathologist. Hence the diagnosis rendered on telepathology consulted specimen should always include a statute of limitation. Histopathology observation is subjective and poorly reproducible. The progress in digitalization of images should continue till it equals which is seen under the standard light microscope. The pathologist's skill in interpreting the digital images will improve till it matches their skills with conventional images.

When telepathology is used for routine workload we need postgraduates and local pathologists who are trained to use the facility to read digital images. If all the postgraduates depend on the telepathology learning solely, there can be damaging loss of local expertise or to their own benefits in light microscopic learning. Acceptance of responsibility by the local pathologist for the final telepathology report becomes a major problem. And if it always becomes the responsibility of others, then the postgraduates will not learn to be very precise and may defer to take the responsibility in future.

Like every new specialty, telepathology also needs legal support. [17] Several complex legal and regulatory issues, such as quality, patient confidentiality, licensing evaluation, practice standards, reimbursement, patient and provider acceptance, equipment standard and compatibility and malpractice will have to be addressed before a widespread use of telepathology can occur. Research into telepathology has been in progress and various formats are available. All have inherent problems, yet interest continues to increase worldwide, with a growing consensus regarding acceptable application, especially those related to quality assurance and teaching. Our present study has confirmed the benefits one can get from telepathology, the evolving medical science. 


\section{REFERENCES}

[1] R. S. Weinstein, A. M. Graham, L. C. Richter, G. P. Barker, E. A. Krupinski, A. M. Lopez, Y. Yagi, J. R. Gilbertson, et al., "Overview of Telepathology, Virtual Microscopy and Whole Slide Imagining: Prospects for the Future," Human Pathology, Vol. 40, No. 8, 2009, pp. 1057-1069. doi:10.1016/j.humpath.2009.04.006

[2] S. Kumar, S. Kumar and B. E. Dunn, Eds., "Telepathology: An Audit,” In: Telepathology, Springer-Verlag, Berlin Heidelberg, 2009, pp. 225-229. doi:10.1007/978-3-540-85786-0_16

[3] R. S. Weinstein, "Prospects for Telepatholgy (Editorial)," Human Pathology, Vol. 17, 1986, pp. 443-434. doi:10.1016/S0046-8177(86)80028-4

[4] K. Kayser, B. Molnar and R. S. Weinstein, "Digital Pathology Virtual Slide Technology in Tissue-Based Diagnosis, Research and Education," VSV Interdisciplinary Medical Publishing, Berlin, 2006, pp. 1-193.

[5] T. Dennis, R. D. Start and S. S. Cross, "The Use of Digital Imaging, Video Conferencing and Telepathology in Histopathology: A National Survey," Journal of Clinical Pathology, Vol. 58, No. 3, 2005, pp. 254-258. doi:10.1136/jcp.2004.022012

[6] B. Stanberry, "Telemediicne: Barriers and Opportunities in the $21^{\text {st }}$ Century," Journal of Internal Medicine, Vol. 247, No. 6, 2000, pp. 615-628. doi:10.1046/j.1365-2796.2000.00699.x

[7] S. A. Wells and C. Sowter, "Telepathology: A Diagnostic Tool for the Millennium?” Journal of Pathology, Vol. 191, No. 1, 2000, pp. 1-7. doi:10.1002/(SICI)1096-9896(200005)191:1<1::AID-PA TH624>3.0.CO;2-S

[8] Y. Shimosato, Y. Yagi, K. Yamagishi, et al., "Experience and Present Status in National Cancer Center Hospital, Tokyo,” Zentralblatt fur Pathologie, Vol. 138, No. 6, 1992, pp. 413-417.

[9] D. S. Winberg, F.-A. Allaert, P. Dusserre, et al., "Telepathology Diagnosis by Means of Still Digital Images: An International Validation Study," Human Pathology, Vol. 27, No. 2, 1996, pp. 111-118. doi:10.1016/S0046-8177(96)90363-9
[10] B. E. Halliday, A. K. Bhatacharya, A. R. Graham, et al., "Diagnostic Accuracy of an International Static-Imaging Telepathology Consultation Service,” Human Pathology, Vol. 28, No. 1, 1997, pp. 17-21. doi:10.1016/S0046-8177(97)90273-2

[11] A. J. Evans, R. Chetty, B. A. Clarke, S. Croul, D. M. Ghazarian; T. R. Kiehl, B. P. Ordonez, S. Ilaalagan, et al., "Primary Frozen Section Diagnosis by Robotic Microscopy and Virtual Slide Telepathology: The University Health Network Experience,” Human Pathology, Vol. 40, No. 8, 2009, pp. 1069-1081. doi:10.1016/j.humpath.2009.04.012

[12] B. E. Dunn, H. Choi, D. L. Recla, S. E. Kerr and B. L. Wagenman, "Robotic Surgical Telepathology between the Iron Mountain and Milwaukee Department of Veterans Affairs Medical Centers: A 12-Year Experience,” Human Pathology, Vol. 40, No. 8, 2009, pp. 1092-1099. doi:10.1016/j.humpath.2009.04.007

[13] A. R. Graham, A. K. Bhattacharyya, K. M. Scott, F. Lian, L. L. Grasso, L. C. Richter, J. T. Henderson, J. B. Carpenter, et al., "Virtual Slide Telepatholgoy for an Academic Teaching Hospital Surgical Patholgoy Quality Assurance Program," Human Pathology, Vol. 40, No. 8, 2009, pp. 1129-1136. doi:10.1016/j.humpath.2009.04.008

[14] C. Massone, H. P. Soyer, G. P. Lozzi, A. DiStefani, B. Leinweber, G. Gabler, et al., "Feasibility and Diagnostic Agreement in Teledermatology Using a Virtual Slide System,” Human Pathology, Vol. 38, No. 4, 2007, pp. 546-554. doi:10.1016/j.humpath.2006.10.006

[15] F. R. Dee, "Virtual Microscopy in Pathology Education," Human Pathology, Vol. 40, No. 8, 2009, pp. 1112-1121. doi:10.1016/j.humpath.2009.04.010

[16] L. A. Bruch, B. R. De Young, C. D. Kreiter, T. H. Haugen, T. C. Leaven and F. R. Dee, "Competency Assessment of Residents in Surgical Pathology Using Virtual Microscopy,” Human Pathology, Vol. 40, No. 8, 2009, pp. 1122-1128. doi:10.1016/j.humpath.2009.04.009

[17] R. Piece, “Cybermedicine,” New England Journal of Medicine, Vol. 339, No. 9, 1998, pp. 638-639. doi:10.1056/NEJM199808273390920 